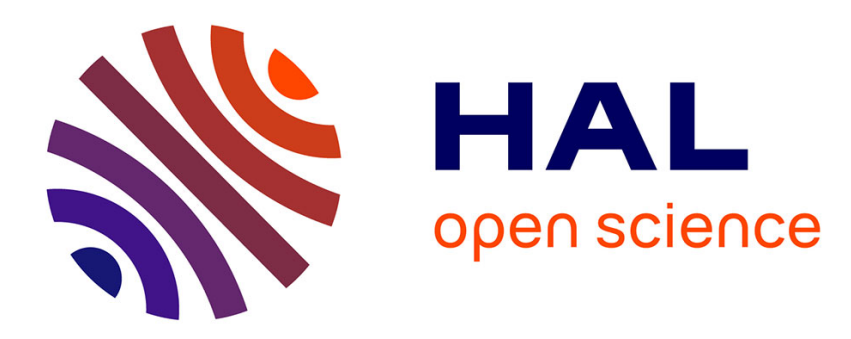

\title{
Longitudinal Vortices in Granular Flows
}

Yoel Forterre, Olivier Pouliquen

\section{To cite this version:}

Yoel Forterre, Olivier Pouliquen. Longitudinal Vortices in Granular Flows. Physical Review Letters, 2001, 86, pp.5886 - 5889. 10.1103/PhysRevLett.86.5886 . hal-01432240

\section{HAL Id: hal-01432240 \\ https://hal.science/hal-01432240}

Submitted on 11 Jan 2017

HAL is a multi-disciplinary open access archive for the deposit and dissemination of scientific research documents, whether they are published or not. The documents may come from teaching and research institutions in France or abroad, or from public or private research centers.
L'archive ouverte pluridisciplinaire HAL, est destinée au dépôt et à la diffusion de documents scientifiques de niveau recherche, publiés ou non, émanant des établissements d'enseignement et de recherche français ou étrangers, des laboratoires publics ou privés. 


\title{
Longitudinal Vortices in Granular Flows
}

\author{
Y. Forterre and O. Pouliquen \\ Institut Universitaire des Systèmes Thermiques et Industriels (IUSTI), 5 rue Enrico Fermi, 13453 Marseille cedex 13, France
}

(Received 20 December 2000; revised manuscript received 6 March 2001)

\begin{abstract}
We present a new instability observed in rapid granular flows down rough inclined planes. For high inclinations and flow rates, the free surface of the flow experiences a regular deformation in the transverse direction. Measurements of the surface velocities imply that this instability is associated with the formation of longitudinal vortices in the granular flow. From the experimental observations, we propose a mechanism for the longitudinal vortex formation based on the concept of granular temperature.
\end{abstract}

DOI: 10.1103/PhysRevLett.86.5886

PACS numbers: 45.70.Mg, 45.70.Qj, 47.32.Cc

In fluid mechanics, flow instabilities lead to the formation of patterns which strongly affect the dynamics, yielding coherent structures and controlling the transition to turbulence [1]. For granular flows, the relevance of these hydrodynamic concepts and the existence of a similar scenario are open issues. Although granular media can exhibit fluidlike properties, they do not behave like classical fluids $[2,3]$. The dissipative nature of grain interactions and the overlap between grain scale and flow scale are fundamental differences with classical fluid flows which lead to difficulties in our research for a hydrodynamic description of granular flows [4]. One way to better understand the specificities of granular flows is to study the instabilities that can develop in these media. Pattern formation has been investigated in vertically vibrated granular layers where the nontrivial balance between the injected energy and the dissipation due to collision leads to a rich phenomenology $[5,6]$. The instability of simple shear flows leading to the formation of clusters has also been studied numerically and theoretically $[7,8]$. In contrast, few studies concern the stability of granular flows along slopes which are the paradigm for geophysical situations. The formation of roll waves has been reported [9] and a fingering instability observed during the propagation of a granular front has been described [10]. In this Letter we present a new instability that spontaneously generates longitudinal vortices (i.e., with axes parallel to flow) in rapid granular flows down rough inclined planes. Although such structures are common in fluid mechanics (Görtler vortices [11,12], streaks in turbulent boundary layers $[13,14])$, they have never been observed in a granular flow. From the experimental observations, we propose a mechanism for the longitudinal vortex formation which is specific to rapid granular flow and relies on the concept of granular temperature [15].

The experimental setup consists of a rough inclined plane with a reservoir containing the granular material (Fig. 1). The plane is a glass plate $(1.3 \mathrm{~m}$ long, $0.3 \mathrm{~m}$ wide) made rough by randomly gluing one layer of particles onto its surface. The glued particles are the same as the ones used for the flow and cover about $70 \%$ of the plane. The side walls are glass plates and have no influence on the flow considering the large width of the channel.
The results presented here have been obtained using sand with a mean diameter $d=0.25 \pm 0.03 \mathrm{~mm}$. Experiments carried out with $0.8 \mathrm{~mm}$ sand and $0.5 \mathrm{~mm}$ monodispersed glass beads give the same results. The amount of particles in the reservoir is always large enough to ensure a constant flow rate during the whole experiment. Therefore, the only two control parameters of the experiment are the inclination of the plane $\theta$ and the opening of the gate $h_{g}$. This configuration has been widely used to study the rheology of granular flows [16-18], instabilities [10], and avalanches dynamics [19], and provides a simple laboratory model for geological granular flows down slopes.

Here we study a new instability observed when the inclination $\theta$ and the opening $h_{g}$ are large (Fig. 2). In this regime, the granular material flowing out from the reservoir accelerates along the slope while the thickness of the granular layer decreases. At a certain distance from the outlet (from 0.4 to $1.3 \mathrm{~m}$ depending on the flow conditions),

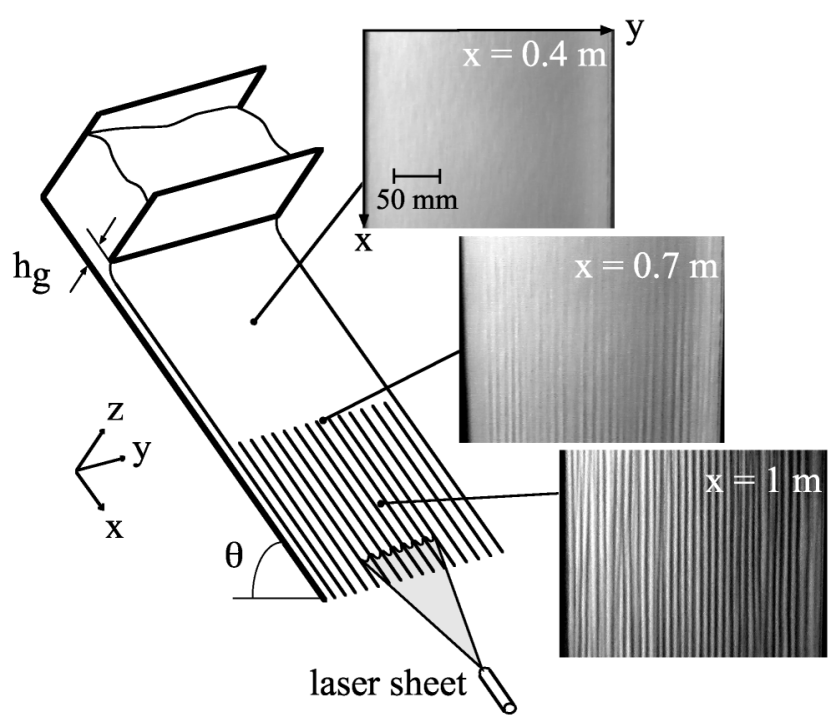

FIG. 1. Sketch of the experimental setup. The three pictures correspond to top views of the free surface lit from the side at three different locations along the slope. $\theta=41^{\circ}, h_{g}=$ $13 \mathrm{~mm}$. The sheet laser light is used to measure the surface deformation (see picture in Fig. 2). 
a regular pattern develops and longitudinal streaks parallel to the flow direction are observed (Fig. 1). In the pictures of Fig. 1, the plane is lit from the side. The bright and dark stripes are thus the signature of a periodic free surface deformation. A sheet laser light projected at a low incident angle provides a precise measurement of the local thickness (Fig. 2). About $0.2 \mathrm{~m}$ downstream of the initiation of the instability, the pattern reaches a "saturated" state: the deformation amplitude $\Delta h$ between crests and troughs and the pattern wavelength $\lambda$ remain constant along the slope. In this saturated state the mean flow also becomes uniform: mean thickness $h$, mean velocity, and mean volume fraction no longer vary along the slope. The wavelength $\lambda$ seems to scale with the mean layer thickness: $\lambda \sim 3 h$. However, it is difficult to significantly vary $h$. In the saturated state, $h$ varies between only 2.1 and $2.6 \mathrm{~mm}$ when varying the height of the gate $h_{g}$ or the inclination $\theta$ over the range indicated in Fig. 2.

The streaked pattern described above is not stationary, but slowly drifts in the transverse $y$ direction with a phase velocity small compared to the chute $x$ velocity (the drift is about $1 \mathrm{~cm} / \mathrm{s}$ while the chute velocity is of the order of $1 \mathrm{~m} / \mathrm{s}$ ). This drift leads to a complex nonlinear spatiotemporal evolution including annihilations and creations of streaks, which is beyond the scope of this study. In this Letter we focus on the flow structure and try to understand the underlying instability mechanism.

We have first investigated the grain motions once the pattern is fully developed, by measuring the velocity field at the free surface. For this purpose, black tracers of the same size of the bulk particles are mixed in the material. The flow being rapid, a fast charge-coupled device (CCD) (charge-coupled device) camera (4500 frames/s, Kodak

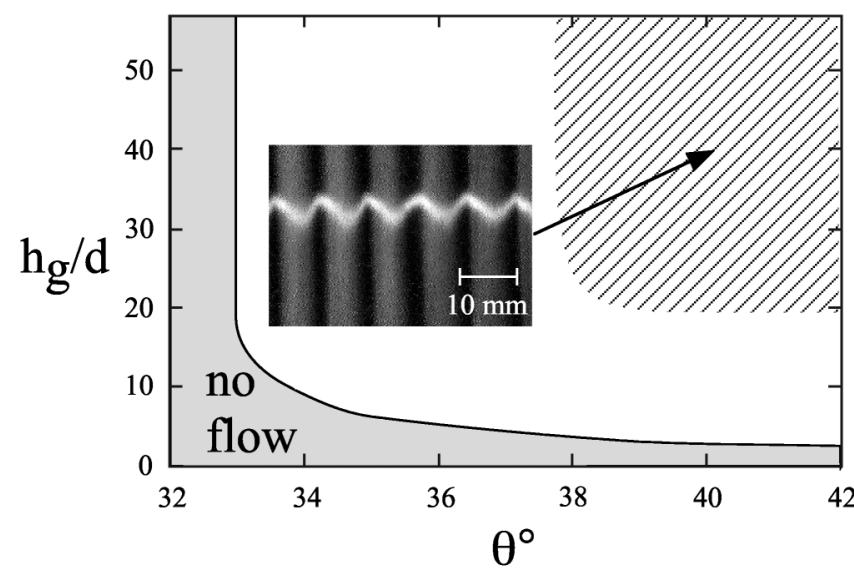

FIG. 2. Phase diagram in $\left(\theta, h_{g}\right)$ plane: the instability is observed in the hatched zone. The picture is a closeup of the free surface in the saturated regime $\left(\theta=41^{\circ}\right.$ and $\left.h_{g}=13 \mathrm{~mm}\right)$. The corrugated laser line provides the thickness profile measurement: mean thickness $h \sim 2.5 \mathrm{~mm}$, deformation amplitude $\Delta h \sim 0.5 \mathrm{~mm}$, wavelength $\lambda \sim 7.5 \mathrm{~mm}$.
HS4540 Ektapro) is necessary to capture the particles displacement at the surface. The movies of particles displacement are analyzed using a particle image velocimetry method [20]. Typical longitudinal and transverse velocity profiles $V_{x}(y)$ and $V_{y}(y)$ are given in Fig. 3a. The instability induces spatial velocity modulations which are correlated with the surface deformation. First, one observes that the longitudinal particle velocity $V_{x}$ is no longer uniform across the bed, but becomes greater in the troughs than in the crests with the modulation of $\Delta V_{x}$ being about $20 \mathrm{~cm} / \mathrm{s}$. The second result is that the instability also induces periodic modulations of the transverse velocity $V_{y}\left(\Delta V_{y} \sim 4 \mathrm{~cm} / \mathrm{s}\right)$ : particles no longer follow the bed slope, but also experience lateral motions. The important observation is the $\pi / 2$ phase shift between $V_{x}$ and $V_{y}$ : in the troughs $V_{x}$ is maximum, whereas $V_{y}$ vanishes. This implies that particles move from the crests towards the troughs as sketched in Fig. $3 \mathrm{~b}$.

This two-dimensional picture of the surface flow implies a three-dimensional particle motion and the presence of longitudinal vortices in the bulk. The mean flow being uniform, mass conservation indeed requires that particles move upwards at the crests and downwards at the troughs. The longitudinal vortices are then counterrotating with one wavelength $\lambda$ of the wavy surface corresponding to a pair of vortices as sketched in Fig. 3b. This 3D structure is reminiscent of patterns observed in liquid flows including Görtler vortices in curved channel flow $[11,12]$ or longitudinal vortices in laminar/turbulent-boundary-layer transition [13,14,21]. However, the Görtler instability is driven by the inertial forces coming from the curvature, while streamwise vortices in boundary layers seem to be linked

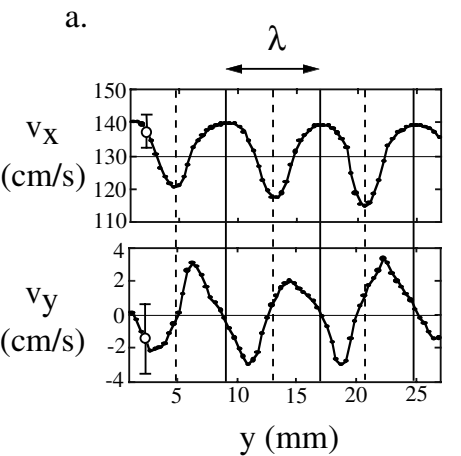

b.

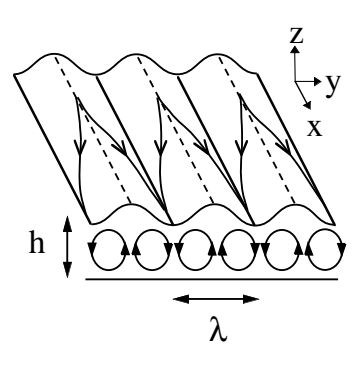

FIG. 3. (a) Longitudinal and transverse free surface velocity profiles $V_{x}(y)$ and $V_{y}(y)\left(\theta=41^{\circ}, h_{g}=13 \mathrm{~mm}\right)$. The vertical solid (respectively, dashed) line indicates the position of troughs (respectively, crests). To get these measurements, a $30 \times 30 \mathrm{~mm}$ area of the free surface (at $x=1.1 \mathrm{~m}$ ) is imaged with a 4500 frames/s fast CCD camera. The PIV method gives the velocity field $V_{x}(x, y)$ and $V_{y}(x, y)$ from two time-successive pictures with a spatial resolution of $0.3 \mathrm{~mm}$. $V_{x}(y)$ and $V_{y}(y)$ are obtained by averaging the velocity field along the $x$ axis and by averaging over 50 successive pictures $(11 \mathrm{~ms})$. The error bars represent the dispersion of the measurements. (b) Sketch of the particule trajectories showing the longitudinal vortices. 
to the turbulent nature of the flow [21]. Therefore, neither of these mechanisms can explain the structure we observed in our granular flow configuration.

To understand the instability mechanism, we have studied how the volume fraction (the ratio between the volume occupied by the particles and the total volume, called simply density in the following) varies in the flow. The first result concerns the mean density measured by trapping some material during the flow and weighing the trapped material. The mean density is found to be never higher than $0.3 \pm 0.05$ in the whole range of parameters where the instability is observed. This result means that the instability takes place in a dilute flow regime. Second, the density is not uniform across the bed when the flow becomes unstable but varies periodically with the surface deformation: crests are dilute and troughs are dense. Experimental evidence of this density variation is presented in Fig. 4a. To obtain this picture, the transparent rough bed is lit from below and the light transmitted through the flow is collected from above by a CCD camera. The light transmitted by the thick part (the crest) is greater than the light transmitted by the thin part (the trough). This is possible only if the crests are more dilute than the troughs.

A complete picture of the flow pattern in a cross section can then be sketched in order to present the surface deformation, the grain motion, and the density (Fig. 4b). One observes that the dense (thus heavy) part of the flow is going down while the dilute (thus light) part is going up, suggesting that density plays an important role in the

a.
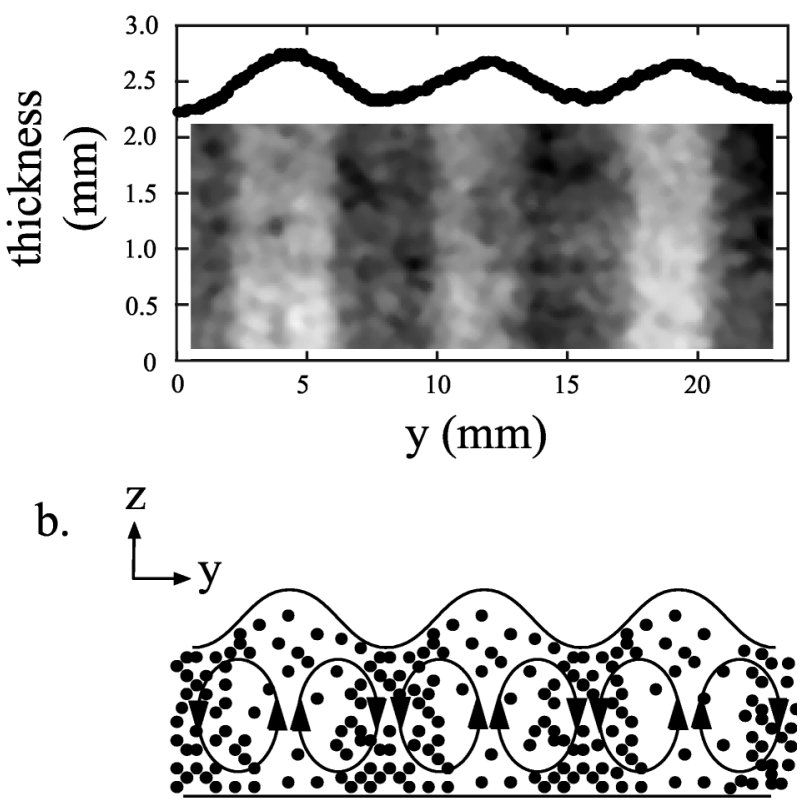

FIG. 4. (a) Picture obtained by lighting the flow from below to obtain information on the local volume fraction. The plots give the corresponding free surface transverse deformation. (b) Sketch of the flow in a cross section. instability. We thus propose the following mechanism to explain the formation of the granular longitudinal vortices. When the instability appears, the flow is rapid and dilute: its dynamics are controlled by the particle-particle and particle-boundary collisions. In this regime, the granular material can be seen as a dissipative dense gas, and a granular temperature can be defined related to the fluctuating motion of the grains [15]. In our flow down a rough inclined plane, the source of the fluctuating motion is the bed roughness. Hence, as the flow accelerates from the outlet of the reservoir, particles close to the plane become more and more agitated due to collisions with the rough bed. The bottom granular temperature then increases along the slope. Consequently, the density at the bottom decreases (as in a molecular gas, density in a granular gas decreases when increasing the temperature) and eventually becomes smaller close to the plane than above. The flow is then mechanically unstable under gravity because the heavy material is above the light one yielding convective longitudinal rolls. There is a close analogy between this situation and the case of a liquid heated from below (Rayleigh-Bénard instability). When a liquid film is flowing down an inclined hot plate, one observes longitudinal convective rolls [22]. However, in this case the heating is provided by the imposed temperature at the bottom plate, whereas in the granular flow, the heating is produced by the flow itself. The rough surface induces a strong shear at the bottom which agitates the material from below.

The mechanism we propose is based on the density profile inversion. In a granular dissipative gas, the density profile actually results from a complex balance between gravity, collisions and dissipation, and its prediction is not straightforward. Density profiles with higher density at the free surface than below have been indeed observed in numerical simulations of rapid flows using discrete element methods $[17,23]$. However, no instability was observed because the simulations were two dimensional. In order to investigate the relevance of the proposed instability mechanism, we have performed a linear stability analysis of steady uniform flows in the framework of the kinetic theory of rapid granular flows [15,24-27]. We have found that inverted density profiles are unstable to transverse perturbations and yield to longitudinal rolls with transverse variations of velocities and density in qualitative agreement with the experimental observations [28].

The new instability presented in this paper leads to a spontaneous pattern formation in a granular flow. An important issue is whether the longitudinal vortices represent the first step in a global scenario leading to more and more complex structures. This evolution towards disordered states is well known in fluid mechanics for the transition to turbulence, but could be strongly affected in granular flows by the dissipative nature of the grain interactions.

This work was supported by the French Ministry of Research and Education (ACI blanche \#2018). We thank the 
Institut de Recherche sur les Phénomènes Hors Equilibre (IRPHE, Marseille) for the use of their fast CCD camera and C. Clanet for his help. Many thanks go to O. Cardoso who provided us with the PIV macro. This work would not have been possible without the technical assistance of F. Ratouchniak.

[1] C. Godrèche and P. Manneville, Hydrodynamics and Nonlinear Instabilities (Cambridge University Press, Cambridge, U.K., 1998).

[2] H. J. Jaeger, S. R. Nagel, and R. P. Behringer, Rev. Mod. Phys. 68, 1259 (1996).

[3] J. Rajchenbach, Adv. Phys. 49, 229 (2000).

[4] I. Goldhirsch, Chaos 9, 659 (1999).

[5] S. Douady, S. Fauve, and C. Laroche, Europhys. Lett. 8, 621 (1989).

[6] P. B. Umbanhowar, F. Melo, and H. L. Swinney, Nature (London) 382, 793 (1996).

[7] S. B. Savage, J. Fluid Mech. 241, 109 (1992).

[8] M. Alam and P. Nott, J. Fluid Mech. 377, 99 (1998).

[9] S. B. Savage, in Theoretical and Applied Mechanics, edited by P. Germain, M. Piau, and D. Callerie (Elsevier, Amsterdam, 1989).

[10] O. Pouliquen, J. Delour, and S. B. Savage, Nature (London) 386, 816 (1997).

[11] H. Görtler, Nachr. Ges. Wiss. Göttingen, New Ser. 2, No. 1 (1940).

[12] W. S. Saric, Annu. Rev. Fluid Mech. 26, 379 (1994).

[13] P. S. Klebanoff, K. D. Tidstrom, and L. M. Sargent, J. Fluid Mech. 12, 1 (1962).
[14] Y. S. Kachanov, Annu. Rev. Fluid Mech. 26, 411 (1994).

[15] C. S. Campbell, Annu. Rev. Fluid Mech. 22, 57 (1990).

[16] S. B. Savage, in Advances in Applied Mechanics, edited by T. Y. Wu and J. Hutchinson (Academic Press, London, 1984).

[17] E. Azanza, F. Chevoir, and P. Moucheront, J. Fluid Mech. 400, 199 (1999).

[18] O. Pouliquen, Phys. Fluids 11, 542 (1999).

[19] A. Daerr and S. Douady, Nature (London) 399, 241 (1999).

[20] O. Cardoso, D. Marteau, and P. Tabeling, Phys. Rev. E 49, 454 (1994). The PIV program is based on the public domain NIH image program, developed by W. Rasband at the U.S. National Institutes of Health and available from the Internet at http://rsb.info.nih.gov/nih-image/. Both adapted source code and compiled application for PIV on MacOS have been developed by O. Cardoso from LBHP-Université Paris 7, and are available at http://134.157.79.91/olivier/NIH/NIH.html.

[21] Y. Aihara, J. Fluid Mech. 214, 111 (1990).

[22] E. M. Sparrow and R. B. Husar, J. Fluid Mech. 37, 251 (1969).

[23] C. S. Campbell and C. E. Brennen, Trans. ASME, J. Appl. Mech. 52, 172 (1985).

[24] C. K. K. Lun, S. B. Savage, D. J. Jeffrey, and N. Chepurniy, J. Fluid Mech. 140, 223 (1984).

[25] J. J. Brey, J. W. Dufty, C. S. Kim, and A. Santos, Phys. Rev. E 58, 4638 (1998).

[26] N. Sela and I. Goldhirsch, J. Fluid Mech. 361, 41 (1998).

[27] J. T. Jenkins and M. W. Richman, J. Fluid Mech. 171, 53 (1986)

[28] Y. Forterre and O. Pouliquen, "Stability Analysis of Rapid Granular Chute Flows: Formation of Longitudinal Vortices" (to be published). 